\title{
Exposition à court terme à la pollution de l'air en ville : apports et limites des différents types de modèles d'estimation de la pollution
}

\section{Gilles Maignant}

\section{(2) OpenEdition}

\section{Journals}

Édition électronique

URL : http://journals.openedition.org/rfst/735

DOI : $10.4000 /$ rfst.735

ISSN : 2492-3672

Éditeur

Espaces et SOciétés (UMR 6590)

Référence électronique

Gilles Maignant, « Exposition à court terme à la pollution de l'air en ville : apports et limites des différents types de modèles d'estimation de la pollution », Revue francophone sur la santé et les territoires [En ligne], Miscellanées, mis en ligne le 17 juillet 2017, consulté le 06 avril 2021. URL : http:// journals.openedition.org/rfst/735; DOI : https://doi.org/10.4000/rfst.735

Ce document a été généré automatiquement le 6 avril 2021.

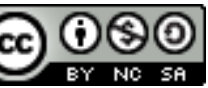

La Revue francophone sur la santé et les territoires est mise à disposition selon les termes de la Licence Creative Commons Attribution - Pas d'Utilisation Commerciale - Partage dans les Mêmes Conditions 4.0 International. 


\title{
Exposition à court terme à la pollution de l'air en ville : apports et limites des différents types de modèles d'estimation de la pollution
}

\author{
Gilles Maignant
}

\section{Introduction}

1 L'organisation mondiale de la santé a reconnu la pollution de l'air extérieur comme une des causes du cancer (WHO, 2013). Sans nier que la pollution de l'air n'est pas absente des zones rurales qui peuvent avoir des concentrations importantes de polluants notamment en pesticides ou encore en ozone, nous nous intéressons dans cet article à la pollution de l'air extérieur en ville, sa connaissance fine en vue d'estimer l'exposition des populations au plus proche de leurs pratiques quotidiennes.

2 De très nombreuses études épidémiologiques s'intéressent aux liens entre concentrations journalières moyennes de polluants, toutes sources confondues et mortalité, par exemple Atkinson montre une corrélation positive entre les concentrations quotidiennes à deux jours en carbone élémentaire et Black carbone et les données de mortalité respiratoire (Atkinson, 2015). Bien qu'intéressante cette étude reste limitée car elle ne permet pas de prendre en compte des données de pollution à échelle fine et la santé différenciée des personnes (on ne connait pas le lieu de vie, ni l'historique de santé des personnes décédées). De même, beaucoup d'études ont montré des liens entre exposition à court terme aux particules fines et risques sur la santé, notamment maladies cardiaques (Dominici et al, 2006 ; Bell et al, 2013). Dans leur métaanalyse, Shah et al. ont trouvé une relation positive, plus forte que pour les autres polluants, entre exposition à court terme aux $\mathrm{PM}_{2.5}$ et insuffisance cardiaque $(\mathrm{RR}=2,12$; $95 \%$ IC $=[1,42 ; 2,82])$, de même pour les $\mathrm{PM}_{10}(\mathrm{RR}=1.63 ; 95 \% \mathrm{IC}=[1,20 ; 2,07])$ (Shah et al., 2013). 
3 Afin d'estimer l'exposition au plus proche de l'individu, nous questionnons les méthodes permettant d'appréhender la pollution de l'air extérieur en ville à échelle fine.

4 En ville, la pollution de l'air présente des différenciations spatiales importantes : des axes à fort taux de pollution peuvent être situés à proximité d'axes à faible taux de pollution (pollution de proximité), toutes choses égales par ailleurs, ce qui explique que des techniques d'interpolations spatiales, peuvent s'avérer inefficaces, rendant la modélisation spatiale incontournable (Chaix, 2009; Maignant, 2010), particulièrement dans le cadre où le réseau de surveillance de la qualité de l'air comptabilise peu de stations ou ne mesure pas les mêmes polluants.

\section{Exposition à la pollution de l'air en ville}

5 L'exposition des populations à la pollution de l'air en ville peut se concevoir à différentes échelles de temps et d'espace, que ce soit du point de vue des concentrations de polluants (sur une agglomération, un quartier ou une rue, en temps réel ou moyennes journalières ou annuelles), ou du point de vue des entités sociales exposées (individu, groupe, sur un court ou long terme).

6 En effet, de très nombreuses études nationales et internationales traitent de cette question. Ainsi, Denis Bard et al. ont étudié les relations entre l'exposition à court terme à la pollution atmosphérique ambiante, le statut socio-économique des populations et la survenue de l'infarctus du myocarde dans la Communauté Urbaine de Strasbourg (CUS, Bas-Rhin) (Bard, 2012). L'exposition estimée dans ce cas, est une exposition moyenne calculée à l'IRIS basée sur des concentrations. Tenailleau, quant à lui, questionne la relation qu'il existe entre la définition d'un voisinage et l'évaluation de l'exposition (Tenailleau, 2015). Gauvin et al. ont montré que l'exposition individuelle à la pollution de l'air et les mesures ambiantes de pollution de l'air sont peu corrélées à court terme bien que les mesures produites par les différents réseaux de surveillance, soient celles utilisées pour caractériser l'exposition dans les études épidémiologiques (Gauvin, 2002). Ainsi son équipe a couplé des concentrations de $\mathrm{NO}_{2}$ et de particules fines (en air ambiant) pour quatre grandes villes françaises (Grenoble, Nice, Toulouse, Paris) avec des données de trafic pour montrer que les enfants (dont la mobilité journalière est faible, due à leurs activités scolaires) sont plus exposés lorsque le lieu d'activité est proche d'une artère à fort trafic. Cette étude tenait également compte du temps passé dans chacun des lieux d'activités (expositions moyennes). L'exposition des individus dépend, bien évidemment de la concentration en polluants obtenue soit par des mesures soit par des modèles, mais également de paramètres individuels comme les habitudes de vie, les lieux d'activités et de résidences, la mobilité, le statut socioéconomique (Chaix et al, 2009 ; Smith et al, 2010). L'exposition repose sur la qualité de l'estimation des éléments de la chaine « émissions - immissions - expositions ».

\section{Différents types de modèles de pollution urbaine}

7 Quelle que soit l'échelle considérée, il y a deux approches complémentaires pour modéliser la qualité de l'air. La première est statistique et s'appuie sur les différentes mesures effectuées par les réseaux de surveillance de la qualité de l'air, les campagnes 
de mesures faites par les chercheurs, elle procède par cartographie et interpolation. Monitorer l'environnement urbain passe bien évidemment par la collecte des données de stations des différents réseaux de mesure mais également par la mise en place de techniques de géostatistiques (interpolation) ou de pseudo-stations. Les «pseudostations et les stations virtuelles ${ }^{1}$ " permettent de densifier le réseau des points nécessaires à la modélisation, là où l'information est absente; et par conséquent, de mieux cartographier la répartition spatiale des polluants atmosphériques (Ung et al, 2001). Bien souvent, les différentes agences agréées de surveillance de la qualité de l'air fonctionnent par des méthodes d'interpolation spatiale, là où la densité de mesures est suffisamment conséquente (cas des grandes et très grandes agglomérations : Paris, Lyon notamment, avec des modèles statistiques).

8 Sans remettre en cause la pertinence des modèles statistiques, nous nous intéressons ici à la seconde approche qui consiste à l'utilisation de différents modèles déterministes qui permettent de rendre compte de la pollution de l'air extérieur en tout point de l'espace considéré. Cette deuxième approche est très utilisée par les physiciens et chimistes, et est basée sur des équations physiques. En effet, pour des raisons techniques et financières, il est impossible de positionner des stations de mesures en tout point de l'espace. La modélisation doit donc s'appuyer sur les données produites par ces stations mais doit permettre de compléter cette information en tout point de l'espace, particulièrement dans les zones peu denses en stations. Cependant, avec l'arrivée d'outils informatiques de plus en plus puissants, ces agences se sont tournées vers des outils plus complexes de modélisation spatiale de la pollution (modèles déterministes) permettant d'appréhender des phénomènes particuliers comme celui des rues canyon (rues propices à l'accumulation de polluants) ou encore de compléter l'information des différentes stations.

9 Ces modèles peuvent se classer en différentes catégories (gaussien, lagrangien, ou eulérien) selon qu'ils considèrent ou pas, avec plus ou moins de détail, la complexité de la morphologie urbaine qui joue un rôle majeur dans la dispersion des polluants. La qualité des modèles déterministes dépend également de l'échelle du modèle et de sa résolution spatiale (Nieuwenhuijsen, 2003). Des auteurs comme Tenailleau ont montré que, dans une approche déterministe, les modèles doivent à minima tenir compte des sources de pollution, de la topographie, de la morphologie urbaine, et de la météorologie (Tenailleau et al, 2015). En effet, nombreuses sont les études qui montrent que la qualité de l'air en ville est corrélée à la morphologie urbaine (Bechle et al., 2011 ; Clark et al., 2011 ; Hankey et al., 2012).

10 Dans un premier temps nous distinguerons les modèles déterministes par grande famille (Gaussien, Lagrangien ou Eulérien), avant de s'intéresser à la question de l'échelle spatio-temporelle optimale permettant de prendre en compte les différenciations spatiales à l'intérieur même des villes.

11 Les modèles gaussiens reposent sur une idée simple: la pollution est inversement proportionnelle à une puissance de la distance, la puissance permettant de tenir compte de la rugosité du sol (relief simplifié). Ils sont utilisés dans le cas de la modélisation de la dispersion d'une source fixe que ce soit de manière continue (usines d'incinération) ou d'épisode de pollution exceptionnel ou ponctuel comme par exemple dans le cadre de l'usine d'AZF à Toulouse. Ils ont l'avantage de fournir un résultat très rapidement. Pour que ceux-ci fonctionnent, il faut que les conditions météorologiques (vent, température, stabilité) soient homogènes sur le domaine d'étude (contrainte 
forte). Les hypothèses de calculs étant très simplifiées (quantité de polluants, vitesse et direction d'émission), ces modèles ne permettent pas de prendre en compte les situations de vents faibles (qui sont les vents « polluants »), ni la présence de bâtiments qui induisent des écoulements cisaillés (turbulence). Aussi, leur utilisation en milieu urbain reste très limitée, mais néanmoins ils sont mobilisés lorsqu'une simulation doit être produite dans un laps de temps très court (décision urgente à prendre).

Les modèles lagrangiens à particules constituent une approche différente. Pour faire fonctionner ce type de modèle, il est nécessaire d'avoir un préprocesseur météorologique permettant de modéliser l'écoulement atmosphérique en trois dimensions (champs de vent tridimensionnel). Dans l'approche lagrangienne on suit le mouvement d'une particule ou d'un flot de particules. Le temps de calcul du modèle est proportionnel au nombre de particules suivies dans le domaine de modélisation, ce qui limite à la fois la surface d'étude considérée (de l'ordre de la centaine de mètres au kilomètre) et le nombre de particules à considérer. Ces modèles permettent de suivre la diffusion d'un panache sur une distance assez importante, mais également de caractériser la pollution urbaine si la morphologie du bâti est relativement homogène et que la topographie n'est pas trop accidentée. Ils sont utilisés pour suivre la pollution transfrontière ou encore le suivi de nuages spécifiques comme le nuage de cendres $d u$ volcan islandais (éruption de l'Eyjafjöll, 2010).

Dans les modèles eulériens, l'espace est découpé en mailles élémentaires (type "éléments finis ») (figure 1) sur lesquelles sont résolues les équations de la mécanique des fluides (écoulement et dispersion) en trois dimensions (équations de NavierStockes). Ces modèles permettent de prendre en compte l'hétérogénéité spatiale des champs météorologiques influencés par la topographie et ou la présence de bâtiments. Dans ce type de modèles, qui permettent de prendre en compte la canopée urbaine, le temps de calcul est proportionnel au nombre de mailles et est indépendant du nombre de particules suivies, ce qui permet de suivre un panel de polluants important. A la différence des modèles lagrangiens pour lesquels on suit la particule, l'objectif est différent dans les modèles eulériens. En effet, on comptabilise le nombre (ou la masse) de particules qui traverse la maille élémentaire, ce qui est beaucoup plus intéressant pour la modélisation de la diffusion de polluants issus de sources mobiles comme celles provenant $\mathrm{du}$ trafic automobile. Ces modèles sont très utiles pour la modélisation du calcul des immissions ${ }^{2}$ en ville car d'une part ils permettent de considérer les réactions chimiques ou photochimiques des polluants; d'autre part de tester des configurations spatiales nouvelles ou d'estimer l'impact d'une mesure de réduction de la circulation et faire ainsi de la prospective territoriale. Par exemple, il est possible d'estimer l'impact de l'installation d'une zone trente, de l'élargissement d'une voie ou du remplissage d'une dent creuse de la morphologie. Dans le cadre d'une simulation de dispersion de polluants par un modèle eulérien, le calcul est réalisé de manière itérative : à chaque pas de temps, les équations de la mécanique des fluides sont résolues pour chaque maille du domaine (figure 2); la simulation s'arrête lorsque la différence de valeurs entre deux pas de temps successifs (itérations) pour chacune des mailles du domaine est inférieure à une valeur fixée au préalable (critère de stabilité fixé à 10-6 dans nos simulations), critère préférable à un critère temporel qui ne garantit pas la convergence du modèle. 
Figure 1 : Exemple de modélisation eulérienne d'un quartier de Nice

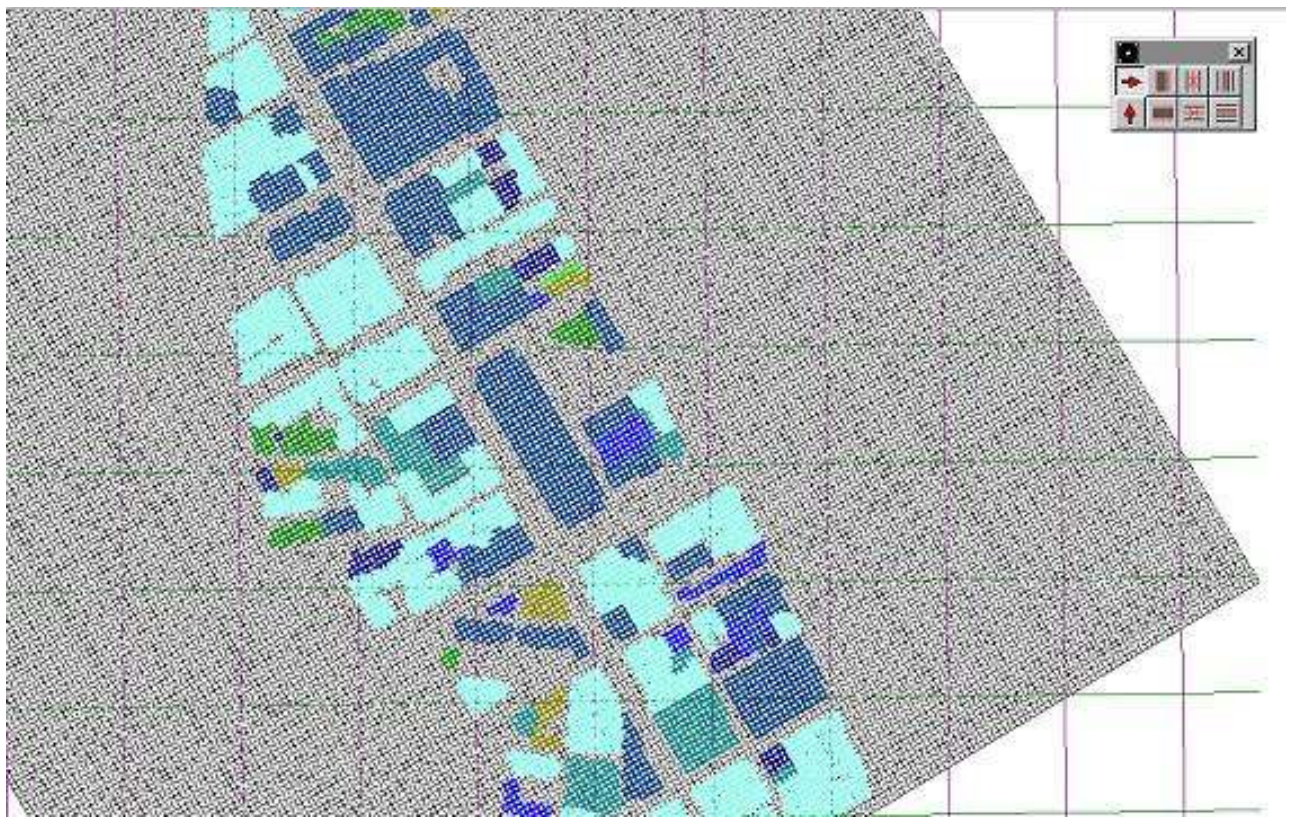

Les différentes couleurs correspondent aux hauteurs différenciées des bâtiments, mesurées au faitage des toits. Le modèle s'appuie sur des données de trafic automobile et de morphologie urbaine permettant, d'une part d'estimer les émissions produites et d'autre part de calculer les immissions résultantes. L'orientation de la rue choisie est NO-SE, sa proximité à la mer est notamment prise en compte par la modélisation en 3D des champs de vents.

Figure 2 : Comparatif des 3 grands types de modèles déterministes.

\begin{tabular}{|c|c|c|c|}
\hline Modèles & Gaussien & Lagrangien & Eulérien \\
\hline $\begin{array}{l}\text { Prise en compte de la } \\
\text { topographie }\end{array}$ & NON & OUI & OUI \\
\hline Météorologie & $\begin{array}{l}\text { Vitesse à } \\
\text { l'émission }\end{array}$ & Processeur météo & $\begin{array}{c}\text { Résolution des } \\
\text { équations de } \\
\text { transport }\end{array}$ \\
\hline Avantages & Rapide & $\begin{array}{l}\text { Prise en compte de la } \\
\text { topographie }\end{array}$ & Physique résolue \\
\hline Inconvénients & $\begin{array}{l}\text { Physique } \\
\text { faible }\end{array}$ & $\begin{array}{l}\text { Nombre de particules } \\
\text { à suivre }\end{array}$ & $\begin{array}{l}\text { Temps de calcul très } \\
\text { long }\end{array}$ \\
\hline $\begin{array}{l}\text { Distance à la source } \\
\text { émettrice }\end{array}$ & $150 \mathrm{~m}$ & $10 \mathrm{~m}$ & $1 \mathrm{~m}$ \\
\hline
\end{tabular}

14 L'un des modèles les plus utilisés dans les études européennes, en accord avec les recommandations de l'OMS est le modèle déterministe eulérien intitulé ADMS - URBAN qui permet de modéliser les concentrations sur plusieurs échelles, allant de l'agglomération à la rue. Dans les travaux de Denis Bard sur l'exposition, la modélisation de la pollution atmosphérique, a également été réalisée à l'aide du modèle ADMS - URBAN permettant de produire des cartes d'immissions de $\mathrm{PM}_{10}, \mathrm{O}_{3}, \mathrm{CO}, \mathrm{NO}_{2}$, et $\mathrm{SO}_{2}$ avec une résolution fine, celle de l'IRIS (Bard, 2012). Le modèle que nous considérons pour tester la sensibilité d'une prise en compte simplifiée ou pas de la morphologie urbaine dans le calcul des immissions est également un modèle déterministe, eulérien à l'échelle de la rue, à savoir MISKAM, qui est utilisé dans de très nombreuses AASQA (agences agréées de la surveillance de la qualité de l'air en France). 
Le terme eulérien signifie que le modèle résout les équations de la mécanique des fluides pour chaque maille élémentaire du modèle.

Ainsi les travaux de Tenailleau et al. s'appuyant sur 800 mesures ont permis de montrer que la distance entre les immeubles et la route est positivement corrélée à la différence d'exposition mesurée à $50 \mathrm{~m}$ et à $400 \mathrm{~m}$ (Tenailleau et al., 2015). Ceci renvoie à la question de l'indice de Landsberg (rue canyon), définissant le gabarit de la rue (hauteur de bâti sur largeur de la rue) permettant de tenir compte des phénomènes d'accumulation de polluants (rues propices aux pièges à polluants).

Dans les deux modèles que nous expérimentons ci-après (modèle simple versus modèle complexe), il s'agit de tester, sur une configuration spécifique de rue canyon, l'impact d'une représentation simplifiée de la morphologie urbaine par rapport à une représentation détaillée de la morphologie urbaine.

17 Comme dans de très nombreux travaux de modélisation (Tenailleau et al, 2015; Maignant, 2010) la taille de maille retenue est $2 * 2 \mathrm{~m}$, horizontalement, permettant d'obtenir une définition fine de la pollution de l'air en lien avec l'objectif espéré (obtenir des concentrations à échelle très fine dans le but d'attribuer à tout point de l'espace urbain une valeur en polluants) et ainsi d'avoir une documentation sur la variation spatio-temporelle de la pollution permettant ensuite de coupler cette information aux itinéraires.

\section{Modèle simple ou modèle complexe pour produire des cartes de concentrations en temps réel}

18 Afin d'étudier la faisabilité d'un modèle d'estimation des concentrations de polluants en temps réel, nous considérons deux modèles d'une même rue : un modèle simple et un modèle complexe, dont la différence résulte dans la prise en compte plus ou moins détaillée de la morphologie urbaine. Ce paragraphe a pour objectif de comparer les résultats entre ces deux modèles, afin de proposer un regard critique sur la modélisation (avantages et limites de chacun) et surtout l'usage qui peut en être fait.

19 Nous l'avons vu, le temps de calcul d'un modèle eulérien est proportionnel au nombre de mailles considérées et inversement proportionnel à la taille de celles-ci $(T=\alpha N / \varepsilon$ où $\mathrm{N}$ est le nombre de mailles et $\varepsilon$ la taille de la maille) mais également dépendant de la surface à modéliser. Autrement dit, si l'on souhaite estimer la pollution à une échelle fine sur un pas de temps court (un, deux ou trois jours), il faut trouver le nombre optimal de mailles ainsi que la finesse de restitution de la morphologie urbaine (quels détails du bâti faut-il sélectionner : les cours intérieures, les porches, les balcons ? et avec quels degrés de précision?) Pour diminuer le temps de calcul ou pour tenir compte de spécificités urbaines particulières, il est possible dans les modèles eulériens de resserrer les mailles sur une zone spécifique présentant un intérêt environnemental certain, tout en agrandissant la taille de maille sur le reste du domaine considéré, c'est le choix fait pour le modèle complexe.

20 Pour illustrer ce propos, nous comparons les résultats des deux modèles eulériens avec des campagnes de mesures effectuées sur le terrain (la rue Bonaparte à Paris où une station du réseau AIRPARIF est présente servira d'illustration du propos). La rue Bonaparte, située dans le $6^{\text {ème }}$ arrondissement de Paris, est une rue canyon. Elle possède une station de mesure à la hauteur de l'école des Beaux-arts (station qui a servi à 
valider les modèles). Pour tester les différences de résultats d'un modèle simple et d'un modèle complexe, nous faisons tourner un modèle tridimensionnel eulérien appelé MISKAM. La troisième dimension correspond à la verticalité et permet de tenir compte du rôle de la morphologie urbaine (bâtiments en trois dimensions) dans la production d'immissions à différentes hauteurs au sein de la rue (gradient vertical d'exposition). Dans ce type de modèle, l'espace bâti est redécoupé en mailles élémentaires sur lesquelles les équations d'advection ${ }^{3}$ - diffusion sont résolues.

21 Dans les deux modèles, tous les paramètres (répartition modale du trafic routier, émissions, climatologie) sont les mêmes, seule la prise en compte détaillée de la morphologie urbaine est différente. Pour le modèle simple, on simplifie au maximum la morphologie urbaine (figure 3) en s'affranchissant des détails comme les cours intérieures, les balcons etc. et en considérant que la morphologie urbaine est rectiligne, juste guidée par la trame viaire. La grille considérée est donc simplifiée avec des mailles équidistantes afin de limiter le temps de calcul. Des essais sur une grille à maille variable ont montré que les résultats sont inchangés, seul le temps de calcul est plus faible.

22 Dans le cadre du modèle complexe, les décrochés de façades, les cours intérieures sont prises en compte pour la simulation (figure 4). La grille est resserrée au niveau de la rue afin d'améliorer la qualité de la simulation tout en n'augmentant pas le temps de calcul de manière trop importante. Comme pour le modèle simple, les émissions sont rentrées dans le modèle de dispersion à partir d'une base de données trafic (nombre de véhicules, part modale, estimation des bouchons...), les conditions météorologiques proviennent de la station située dans le parc de Montsouris (14 ${ }^{\text {ème }}$ arrondissement), station dite de fond. Le pas de temps est horaire (données de trafic automobile, données météorologiques...). La campagne de mesures (mât météorologique) a duré 3 jours ( 4 au 7 décembre) pour l'ensemble de la journée. 
Figure 3 : Modèle simple de la morphologie urbaine de la rue Bonaparte à Paris, pour lequel chaque carré représente l'emprise spatiale horizontale de la maille du modèle (3D)

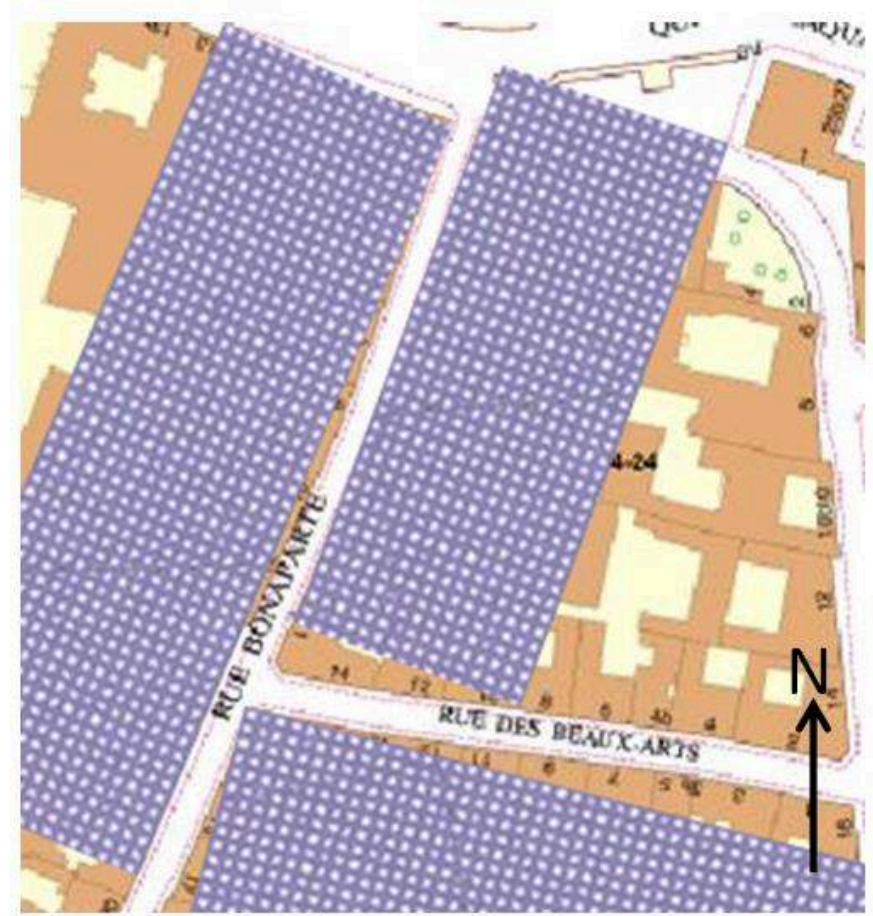

Dans le modèle, le vent est initialisé dans la zone non perturbée par la morphologie urbaine, c'est-à-dire au-dessus des toits (200 mètres), le modèle eulérien qui est un modèle non-hydrostatique permet alors de reconstituer le champ de vent en tout point de n'importe quelle rue, qu'elle soit une rue canyon ou pas. Comme dans tous les modèles non-hydrostatiques, qui sont des modèles itératifs, un critère d'arrêt doit être établi, nous avons opté, pour un critère numérique égal à 10-6. Autrement dit, les simulations s'arrêtent dès que pour chaque maille du modèle, la différence entre deux valeurs successives du paramètre considéré (itérations) est inférieure à cette valeur numérique. 
Figure 4 : Modèle complexe de la morphologie urbaine de la rue Bonaparte à Paris. Les différentes couleurs représentent les différentes hauteurs des bâtiments. Le périmètre du maillage du modèle complexe est le même que celui du modèle simple mais, dans le cadre du modèle simple, il n'est pas nécessaire d'élargir outre mesure la zone considérée au-delà de l'artère considérée (ici la rue Bonaparte) car l'impact de la morphologie sur la dispersion des polluants est très simplifié.

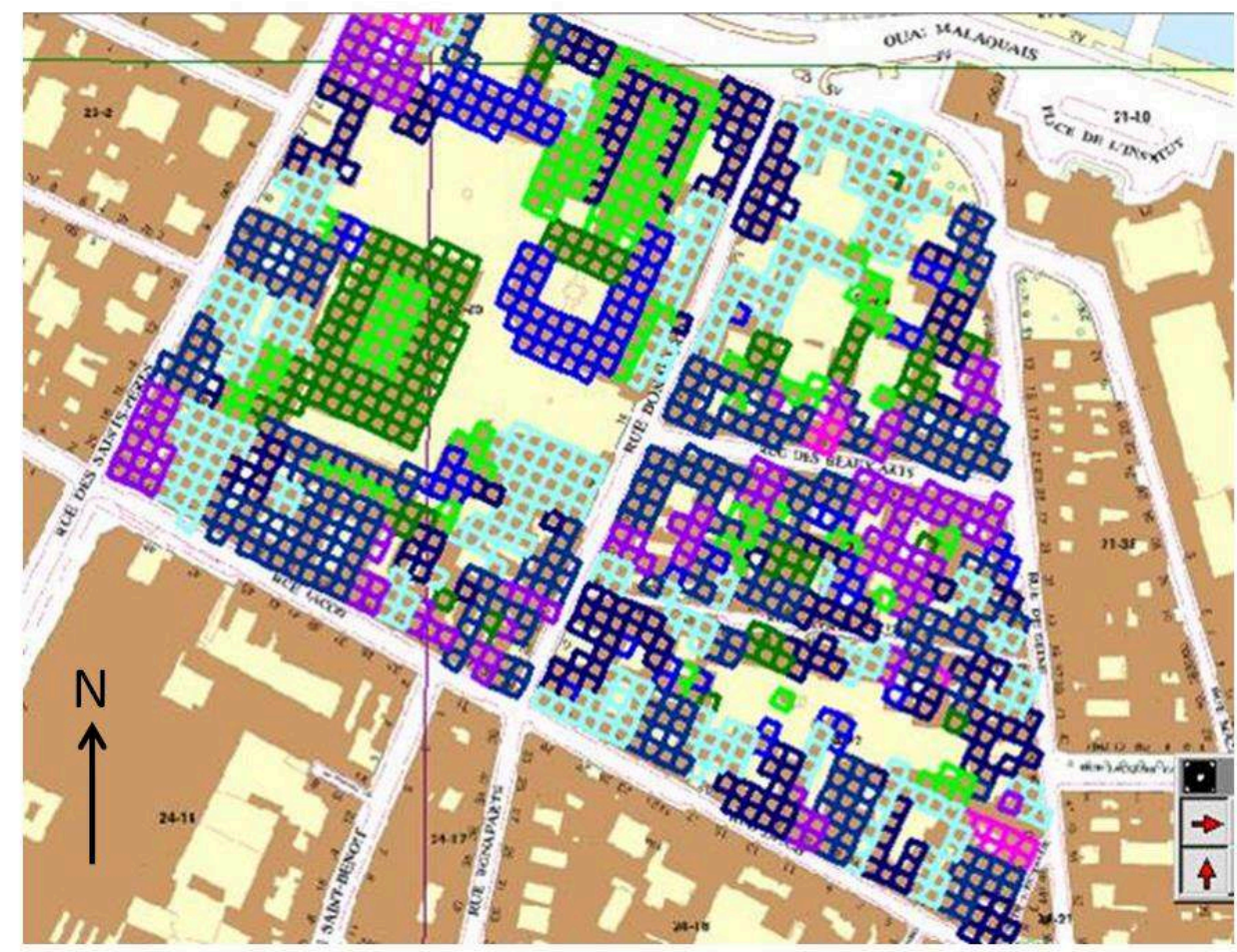

Un mât météo permettant de connaître la vitesse et la direction du vent à l'emplacement de la station de mesures de la pollution a été installé durant toute la campagne de mesures (04-07 décembre 2000). Il permet de comparer les sorties du modèle (vitesse et direction du vent pour chaque maille du modèle, à chaque pas de temps) et les données produites par le mât (figure 5). La comparaison entre les deux modèles se fait au niveau de l'implantation du mât météorologique (vitesse et direction). Les résultats du modèle reflètent bien les données du mât à l'exception de la mesure $7 \mathrm{TU}$ du 4 décembre 2000.

Ainsi, le modèle complexe permet de représenter correctement les champs de vents, à la fois angle et vitesse (résultante) et donc la dispersion des polluants en tout point de l'espace tridimensionnel. Pour de mêmes émissions, la différence entre les concentrations provient des champs de vents qui sont influencés par la morphologie urbaine. L'exposition (c'est-à-dire les immissions) est directement reliée à la qualité d'estimation du champ de vent par le modèle (simple ou complexe). 
Figure 5 : Données des simulations MISKAM faite à partir du modèle complexe (vitesse = Résultante) et direction du vent à la hauteur de mesure du mât, une fois le modèle stabilisé, c'est à dire quand la différence de valeurs entre deux itérations successives est inférieure à 10-6 pour chacune des mailles, il est impossible de comparer les valeurs avant cette stabilisation. Les données de Montsouris servent à initialiser le champ de vent dans le modèle (dans la zone non perturbée au-dessus des toits tandis que le mât sert à valider les sorties de modèle à l'échelle de la rue Bonaparte).

\begin{tabular}{|c|c|c|c|c|c|c|c|c|}
\hline & \multicolumn{2}{|c|}{ Montsouris } & \multicolumn{4}{|c|}{ Simulations MISKAM (modèle complexe) } & \multicolumn{2}{|c|}{ Mat } \\
\hline Dates & Angle $^{\circ}$ & $\begin{array}{l}\text { Vitesse } \\
(\mathrm{m} / \mathrm{s})\end{array}$ & $\begin{array}{c}\text { Moyenne } \\
\text { Composante } \\
\text { horizontale } \\
n^{\circ} 1 \text { du vent } \\
U\end{array}$ & $\begin{array}{l}\text { Moyenne } \\
\text { Composante } \\
\text { horizontale } \\
n^{\circ} 2 \text { du vent } \\
\text { V }\end{array}$ & $\begin{array}{l}\text { Résultante } \\
\text { Modèle } \\
=\text { Vitesse du } \\
\text { vent } \\
\text { modélisée }\end{array}$ & $\begin{array}{l}\text { Angle } \\
\text { Modèle }\end{array}$ & $\begin{array}{l}\text { Angle } \\
\text { Mat }\end{array}$ & $\begin{array}{l}\text { Vitesse } \\
\text { au mat } \\
(\mathrm{m} / \mathrm{s})\end{array}$ \\
\hline $\begin{array}{l}7 \text { TU } 4 \\
\text { Déc }\end{array}$ & 200 & 4 & -0.30 & 1.50 & 1.53 & 193.60 & 211 & 2,2 \\
\hline $\begin{array}{l}8 \text { TU } 4 \\
\text { Déc }\end{array}$ & 180 & 5 & -0.44 & 2.02 & 2.06 & 192.70 & 210 & 1.8 \\
\hline $\begin{array}{l}7 \text { TU } 5 \\
\text { Déc }\end{array}$ & 170 & 4 & -0.35 & 1.51 & 1.55 & 192.02 & 212 & 1,4 \\
\hline $\begin{array}{l}8 \text { TU } 5 \\
\text { Déc }\end{array}$ & 170 & 4 & -0.35 & 1.51 & 1.55 & 192.02 & 211 & 1.3 \\
\hline $\begin{array}{l}7 \text { TU } 6 \\
\text { Déc }\end{array}$ & 190 & 4 & -0.33 & 1.51 & 1.54 & 192.74 & 206 & 1.56 \\
\hline $\begin{array}{l}8 \text { TU } 6 \\
\text { Déc }\end{array}$ & 180 & 4 & -0.35 & 1.53 & 1.57 & 192.20 & 204 & 1.55 \\
\hline $\begin{array}{l}7 \text { TU } 7 \\
\text { Déc }\end{array}$ & 130 & 3 & -0.15 & 0.76 & 0.77 & 193.58 & 207 & 0.9 \\
\hline $\begin{array}{l}8 \text { TU } 7 \\
\text { Déc }\end{array}$ & 130 & 3 & -0.15 & 0.76 & 0.77 & 193.58 & 215 & 1 \\
\hline $\begin{array}{l}9 \text { TU } 7 \\
\text { Déc }\end{array}$ & 130 & 3 & -0.15 & 0.76 & 0.77 & 193.58 & 212 & 0.9 \\
\hline
\end{tabular}

La comparaison des résultats des simulations du modèle simple et du modèle complexe montre une bonne adéquation en termes de comportement (figure 5), en revanche le modèle simple surestime la vitesse du vent (40\%), ainsi que la direction $5 \%$; ainsi ces écarts moyens sont connus et donc quantifiables, cette surestimation vient du fait que le modèle n'est pas capable de prendre en compte les obstacles permettant de freiner les champs de vents. L'avantage du modèle complexe est qu'il permet une meilleure description de la réaliste des concentrations de polluants présents sur chaque artère ; mais il est très gourmand en temps de calcul ( $\left.\mathrm{CPU}^{4}\right)$. Aussi, si l'on souhaite estimer les relations entre environnement et santé, nous devons passer par ce type de modélisation (relation fine). Mais, si on souhaite obtenir un modèle permettant de connaître la pollution de l'air en temps réel (pas de temps d'une minute à une heure), pour ensuite en déduire des expositions fines (horaires), il est nécessaire d'opter pour le modèle simple (figure 8), dont le temps de calcul est réduit, turbulence induite par la morphologie urbaine et donc une mesure plus quitte à faire la correction de la surestimation, compte tenu de la bonne corrélation entre les résultantes $(\mathrm{R})$ des deux modèles (figures 6 et 7). Bien entendu, cet exercice réalisé sur une rue à Paris démontre la faisabilité d'un outil temps réel permettant de connaitre les concentrations à échelle fine et de manière automatisée. 
Figure 6 : Comparaison des résultats des simulations, modèle simple versus modèle complexe U représente la première composante horizontale du vent, $\mathrm{V}$ la seconde composante horizontale du vent, et $R$ la résultante.

\begin{tabular}{|c|c|c|c|c|c|c|c|c|}
\hline & \multicolumn{2}{|c|}{$\begin{array}{l}\text { Simulations } \\
\text { simple) }\end{array}$} & \multicolumn{2}{|c|}{ IISKAM (modèle } & \multicolumn{2}{|c|}{$\begin{array}{l}\text { Simulations } \\
\text { complexe) }\end{array}$} & MISKAM & (modèle \\
\hline Dates & $\mathrm{U}$ & $\mathrm{V}$ & $\mathrm{R}$ & Angle & $\mathrm{U}$ & $\mathrm{V}$ & $\mathrm{R}$ & Angle \\
\hline $\begin{array}{l}7 \text { TU } 4 \\
\text { Déc }\end{array}$ & 0.02 & 2.21 & 2.21 & 204.39 & -0.30 & 1.50 & 1.53 & 193.60 \\
\hline $\begin{array}{l}8 \text { TU } 4 \\
\text { Déc }\end{array}$ & 0.13 & 2.79 & 2.79 & 202.33 & -0.44 & 2.02 & 2.06 & 192.70 \\
\hline $\begin{array}{l}7 \text { TU } 5 \\
\text { Déc }\end{array}$ & 0.13 & 2.16 & 2.16 & 201.55 & -0.35 & 1.51 & 1.55 & 192.02 \\
\hline $\begin{array}{l}8 \text { TU } 5 \\
\text { Déc }\end{array}$ & 0.13 & 2.16 & 2.16 & 201.55 & -0.35 & 1.51 & 1.55 & 192.02 \\
\hline $\begin{array}{l}7 \text { TU } 6 \\
\text { Déc }\end{array}$ & 0.06 & 2.26 & 2.26 & 203.39 & -0.33 & 1.51 & 1.54 & 192.74 \\
\hline $\begin{array}{l}8 \text { TU } 6 \\
\text { Déc }\end{array}$ & 0.10 & 2.24 & 2.25 & 202.45 & -0.35 & 1.53 & 1.57 & 192.20 \\
\hline $\begin{array}{l}7 \text { TU } 7 \\
\text { Déc }\end{array}$ & 0.15 & 0.75 & 0.76 & 193.64 & -0.15 & 0.76 & 0.77 & 193.58 \\
\hline $\begin{array}{l}8 \text { TU } 7 \\
\text { Déc }\end{array}$ & 0.15 & 0.75 & 0.76 & 193.64 & -0.15 & 0.76 & 0.77 & 193.58 \\
\hline $\begin{array}{l}9 \text { TU } 7 \\
\text { Déc }\end{array}$ & 0.15 & 0.75 & 0.76 & 193.64 & -0.15 & 0.76 & 0.77 & 193.58 \\
\hline
\end{tabular}

Figure 7 : Corrélation entre la résultante $\mathrm{R}$ issue du modèle simple et celle du modèle complexe ( 2 modèles), pour les quatre dates de la campagne de mesure de la vitesse du vent et de sa direction au niveau du mât météo.

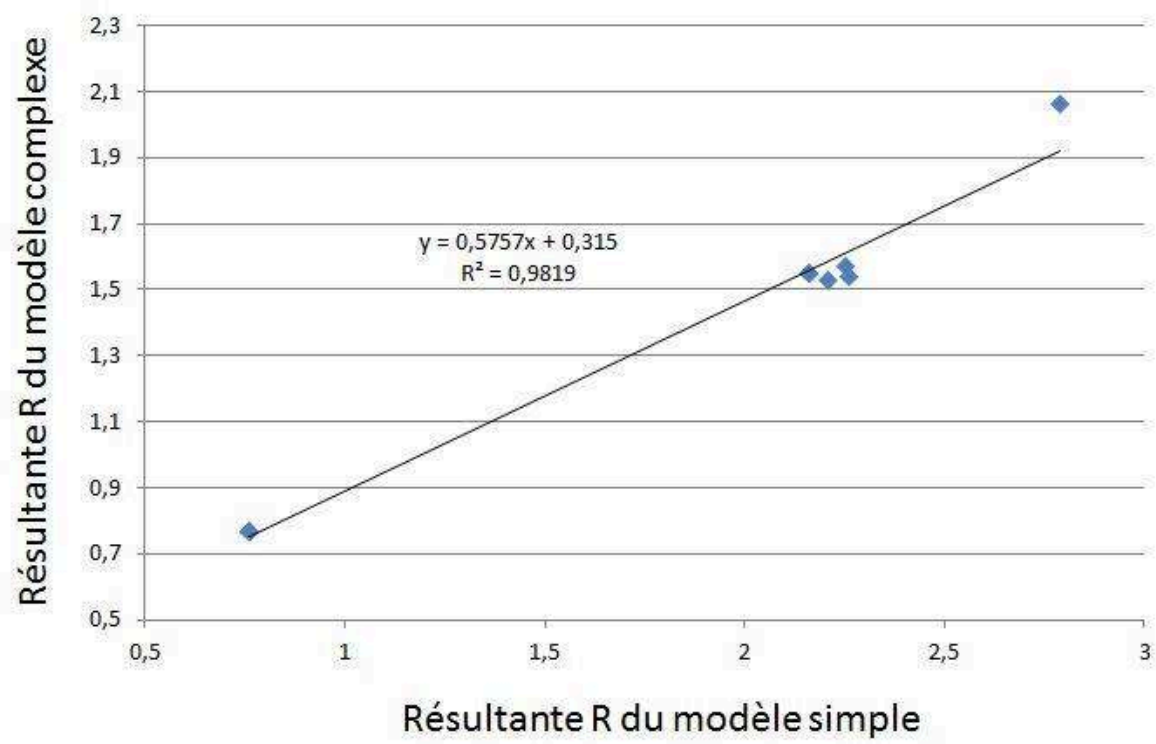


Figure 8 : avantages et inconvénients des deux modèles (modèle simple et modèle complexe)

\begin{tabular}{|c|c|c|}
\hline $\begin{array}{c}\text { Modèle } \\
\text { simpleversus } \\
\text { Modèle } \\
\text { complexe }\end{array}$ & Modèle simple & Modèle complexe \\
\hline Avantages & $\begin{array}{l}\text { Construction du modèle } \\
\text { CPU } \\
\text {. Concentrations bien } \\
\text { modélisées }\end{array}$ & $\begin{array}{l}\text { Meilleure définition de la turbulence } \\
\text { induite par les bâtiments } \\
\text {. Concentrations très bien modélisées }\end{array}$ \\
\hline Inconvénients & $\begin{array}{l}\text { Turbulence mal modélisée } \\
\text {. Champ de vent surestimé }\end{array}$ & . CPU \\
\hline
\end{tabular}

\section{Vers une estimation de l'exposition temps réel : itinéraires individuels d'exposition}

L'air que nous respirons quotidiennement est chargé de polluants dont les effets sur la santé sont complexes et encore partiellement connus, si ce n'est que par des grandes tendances. La pollution respirée tout au long de nos déplacements a été très peu étudiée ou présente quelques fois des résultats contradictoires selon le mode de déplacement utilisé (vélo, marche, course, transports publics, métro, train). L'étude de Good et al, réalisée récemment, est l'une des rares études à s'intéresser à cette exposition différenciée ici, selon nos déplacements et modes de déplacements. Basée sur une cohorte de 45 personnes (Fort Collins) suivies sur 381 jours, l'étude a permis d'estimer l'environnement subi au cours de son déplacement et de voir si des solutions alternatives sont intéressantes, notamment en termes de trajet-temps-exposition. L'étude s'appuie sur un panel de personnes qui ont un trajet domicile-travail d'aumoins $2.4 \mathrm{~km}$, âgées de 18 à 65 ans, titulaires d'un permis de conduire, non-fumeurs et non exposées régulièrement aux poussières et vapeurs professionnelles. Chaque participant devait identifier un trajet direct et des alternatives possibles et répondre à un questionnaire sur leur pratique journalière (chauffage, climatisation, type de véhicule etc.). Des mesures portatives de pollution et d'effort physique étaient effectuées tout au long du trajet, mesures géo-référencées par GPS. La moyenne pondérée dans le temps et l'exposition cumulée à chaque polluant sont ainsi calculées pour chaque trajet (Good et al., 2015).

De fait, on ne connait pas avec précision la pollution de l'air à laquelle sont soumises les différentes populations, de très fortes différenciations spatiales peuvent être présentes au sein des villes, ce qui rend la corrélation pollution - santé très délicate. En effet, on ne connait pas avec précision les différents parcours et pratiques spatiales des individus (environnements successifs, modes de déplacements, statut de fumeurs ou pas, etc.). En ville, différents types d'itinéraires s'offrent au piéton. Plus ou moins consciemment et plus ou moins efficacement, ce dernier va sélectionner son trajet en 
fonction de ses usages. La personne se rendant à son travail aura tendance à privilégier le chemin le plus court en temps, tandis que le promeneur choisira davantage un chemin de bonne qualité environnementale surtout s'il coïncide avec des centres d'intérêts patrimoniaux ou des espaces offerts à la vue. Ce qui montre que l'estimation de l'exposition réelle à la pollution de l'air est complexe à moins de suivre un échantillon de personnes (sentinelles).

La question des itinéraires individuels d'exposition n'est pas nouvelle (Maignant, Dutozia, 2010), mais elle a pris un nouvel essor avec la possibilité d'obtenir grâce aux modèles eulériens (qu'ils soient simples ou complexes) et la puissance de calcul des ordinateurs d'aujourd'hui, une information sur les immissions de polluants en tout point de l'espace à une échelle (spatiale et temporelle) très fine. D'autres approches de modélisation sont également intéressantes à souligner.

Par exemple, les travaux de mesures itinérantes d'ozone sur la ville de Nice, réalisés à vélo par Nicolas Martin, apportent également une connaissance sur la variabilité spatiale de ce polluant et sur l'exposition sous-jacente des habitants. Néanmoins, la représentativité des axes (rues empruntées) reste très limitée car le parcours est toujours le même pour l'ensemble des journées de mesures afin de disposer d'une base de données exploitable statistiquement (Martin, 2009; Martin, 2016). Cette étude a notamment montré que les rues canyons sont moins polluées en ozone, car l'ozone est détruit par réaction chimique avec les autres polluants primaires piégés dans ce type de rue (notamment les monoxydes d'azote). Cette étude a montré que d'une rue à une autre, les concentrations peuvent doubler voire tripler en fonction de la fréquentation des voies de circulation, de la force et de la direction du vent (directement influencées par la morphologie urbaine), du niveau de fond en ozone de la journée, ce qui montre une exposition très différenciée selon le type d'artères (Martin, 2009). De même, Hankey et al. ont estimé l'exposition à vélo à la pollution particulaire (nombre et taille des particules, black carbone et $\mathrm{PM}_{2.5}$ ) en positionnant un capteur sur le vélo parcourant la ville de Minneaopolis dans l'Etat du Minnesota aux Etats-Unis (Hankey et al., 2012), les particules constituant un facteur de risque important pour les maladies cardiovasculaires en milieu urbain (WHO - OMS, 2009). Ainsi les travaux de Hankey se sont appuyés sur une classification des routes empruntées en trois grands types (routes principales, bandes cyclables, sentiers). Les résultats ont montré que les expositions peuvent être très différenciées selon le moment de la journée et la configuration d'artère empruntée, permettant par un aménagement judicieux de faire descendre l'exposition des cyclistes. Mais, les informations éparses de polluants de l'air (comme l'ozone, ou les particules) ne peuvent fournir une source de données suffisante à la quantification de l'exposition à la pollution de l'air extérieur (Sicard, 2016; Kethireddy et al., 2014 ; Araki et al., 2015). Ceci oblige le chercheur ou le technicien des AASQA à avoir recours à des modèles eulériens (type ADMS URBAN ou MISKAM), seule famille de modèles à pouvoir fournir, en tout point de l'espace, les concentrations présentes.

31 Ainsi, de plus en plus de capteurs portatifs arrivent sur le marché. Bien que leur sensibilité ne soit pas toujours bien estimée, ces innovations technologiques actuelles et futures doivent être regardées avec intérêt. Elles permettront sous peu de connaître l'exposition temps-réel des habitants tout au long de leurs itinéraires quotidiens. De nombreuses applications pour Smartphone, géolocalisées, sont en cours de développement. Citons par exemple l'application Ambiciti, développée en partenariat avec NUMTECH qui permet de proposer des cartes horaires de qualité de l'air jusqu'à la 
résolution de la rue, pour une évaluation fine de son exposition. L'application combine toutes les sources d'information disponibles: simulations numériques, observations de capteurs fixes, observations de capteurs mobiles et observation qualitatives. Ainsi, l'avenir repose sur des solutions technologiques impliquant des individus, disposant de capteurs portatifs, capteurs qui pourraient venir compléter les dispositifs des stations fixes.

\section{Conclusion}

Nous l'avons vu, l'estimation précise (mesure) de la pollution de l'air en tout point de l'espace urbain reste encore délicate, de même pour les facteurs influençant l'exposition (vulnérabilité, itinéraires, temps passé sur un axe...). A ces incertitudes de mesures, tant sur les données de pollution que sur les données de santé, se rajoutent des itinéraires personnels distincts, des modes de vie différents qui font que le couplage données de pollution - données de santé reste partiel. Aussi, il faut rester prudent sur les décès qu'il serait possible d'éviter, même si de très nombreuses études cherchent à coupler des données de pollution et des données de santé et plus généralement de mortalité exprimées en facteur de risque (ERPURS, INVS, OMS, ANSES, APHP etc.), Cela ne remet pas en cause l'impact certain de la pollution de l'air sur la santé mais oblige le scientifique a resté prudent sur sa quantification. De très nombreuses études montrent des liens certains entre pollution atmosphérique et impact sur la santé, mais il est nécessaire d'approfondir la connaissance fine de la pollution et de mieux appréhender les parcours individuels (itinéraires de vie, antécédents médicaux, exposition quotidienne différenciée). D'un point de vue méthodologique, Il est de plus nécessaire de mobiliser une approche multifactorielle permettant d'identifier le rôle de chacune des variables dans cette relation complexe environnement-santé. L'échelle géographique de la pollution de l'air et l'échelle des données de santé (que ce soit en termes de mesures ou de réglementations type CNIL) sont souvent incompatibles si ce n'est dans le cas de suivi de cohorte dont les effectifs sont nécessairement réduits (50 à 100 personnes suivies).

\section{BIBLIOGRAPHIE}

ARAKI S, YAMAMOTO K, KONDO A, 2015. Application of regression kriging to air pollutant concentrations in Japan with high spatial resolution. Aerosol Air Qual. Res. 15, 234-241.

ATKINSON R.W, 2015. Short-term exposure to traffic-related air pollution and daily mortality in London, UK. Journal of Exposure Science and Environmental Epidemiology, 1-8

BARD D, HAVARD S, DEGUEN S, PEDRONO G, SCHILLINGER C, EILSTEIN D, SEGALA C, ARVEILER D, 2012. PAISIM : Pollution atmosphérique, inégalités sociales et infarctus du myocarde, $7 \mathrm{p}$.

BECHLE MJ, MILLET DB, MARSHALL JD, 2011. Effects of income and urban form on urban NO2: global evidence from satellites. Environ. Sci. Technol. 45 (11), 4914-4919. 
BELL ML, ZANOBETTI A, DOMINICI F. 2013. Evidence on Vulnerability and Susceptibility to Health Risks Associated With Short-Term Exposure to Particulate Matter: A Systematic Review and Meta-Analysis. American Journal Epidemiology, Vol. $178 \mathrm{n}^{\circ} 6$

CHAIX B, MERLO J, EVANS D, LEAL C, HAVARD S., 2009. Neighbourhoods in eco-epidemiologic research: delimiting personal exposure areas. A response to Riva, Gauvin, Apparicio and Brodeur. Soc SCi. Med. 69, 1306-1310.

CLARK LP, MILLET DB, MARSHALL JD, 2011. Air quality and urban form in the US urban areas: evidence from regulatory monitors. Environ. Sci. Technol. 45 (16), 7028-7035.

CORSO M, PASCAL M, WAGNER V, BLANCHARD M, BLATEAU A, COCHET A et al, 2015. Impact à court terme des particules en suspension (PM10) sur la mortalité dans 17 villes françaises, 2007-2010. Bulletin Epidémiologique Hebdomadaire, (1-2), 14-20. http://www.invs.sante.fr/beh/ 2015/1-2/2015_1-2_3.html

DAB W, ROUSSEL I, 2001. L'air et la ville, Hachette, 219 p.

DOMINICI F, PENG RD, BELL ML, et al. 2006. Fine particulate air pollution and hospital admission for cardiovascular and respiratory diseases.JAMA ; 295 (10), 1127-1134.

GAUVIN S, ZMIROU D, LE MOULLEC Y, CASSADOU S, LAUVERGNE N, REUNGOAT P, VESTRI V, MOMAS I, 2002. Air quality monitoring and personal exposure of children to $\mathrm{NO}_{2}$ and particles, Revue d'épidémiologie de santé publique, Jun ; 50 (3), 307-19.

GOOD N, et al., 2015. The Fort Collins Commuter Study: Impact of route type and transport mode on personal exposure to multiple air polluants. Journal of Exposure Science and Environmental, 1-8.

HANKEY S, MARSHALL JD, BRAUER M., 2012. Health impacts of the built environment within urban variability in physical inactivity, air pollution and ischemic heart disease mortality, Environmental Health Perspectives, 120 (2), 247-253.

HANKEY S, MARSHALL JD, 2015. On-bicycle exposure to particulate air pollution: Particle number, black carbon, PM2.5, and particle size, Atmospheric Environment 122, 65-73.

KETHIREDDY SR, TCHOUNWOU PB, AHMAD HA, YERRAMILLI A, YOUNG JH, 2014. Geospatial interpolation and mapping of tropospheric ozone pollution using geostatistics. Int J. Environ Res Public Health 11, 983-1000.

MAIGNANT G, DUTOZIA J 2010. « Itinéraire des piétons et risques d'exposition en zone urbaine : approche méthodologique », in Sociétés, Environnements, Santé, IRD, pp. 213-235.

MAIGNANT G, 2010. «Pollution de l'air et morphologie urbaine : une relation complexe », Editions Universitaires Européennes, 356 p.

MARTIN N, CARREGA P, 2009. La variabilité spatiale de l'ozone en milieu urbain et périurbain : le cas de Nice, Pollution atmosphérique $\mathrm{n}^{\circ}$ 204, oct-déc, 461-474.

MARTIN N, 2016. Pics de pollution par l'ozone à Nice : comparaison entre 2007 et 2015 des mesures itinérantes et des conditions météorologiques associées, Actes du $29^{\text {ème }}$ colloque de l'association internationale de climatologie, Lausanne-Besançon, 63-68.

NIEUWENHUIJSEN MJ, 2003. Exposure Assesment in Occupational \& Environmental Epidemiology. Oxford University Press.

PERRIN R, 1976. Pollutions et nuisances d'origine industrielle et urbaine dans l'aire métropolitaine marseillaise, Thèse de doctorat, Université Nice Sophia-Antipolis, 697 p. 
ROUSSEL I, 2000. La pollution atmosphérique : risque sanitaire ou risque climatique?

Publications de l'association internationale de climatologie, Vol 13, 538-546.

SBIHI H et al., 2015. Perinatal Exposure to Traffic-Related Air Pollution and Atopy at 1 year of Age in a Multi-Center Canadian Birth Cohort Study. Environmental Health Perspectives, Vol. 123 number 9, 902-908.

SHAH ASV, LANGRISH JP, NAIR H, MCALLISTER DA, HUNTER AL, DONALDDSON K, NEWBY DE, MILLS NL. 2013. Global association of air pollution and heart failure: a systematic review and meta-analysis. Lancet 382, 1039-48.

SMITH G, GIDLOW C, DAVEY R, FOSTER C, 2010. What is my walking neighbourhood? A pilot study of English adults' definitions of their local walking neighbourhoods. Int. J. Behav Nutr. Phys. Act. 7, 34-42.

TARNIER P, 2001. Le scandale de l'air contaminé, Mango, 195 p.

TENAILLEAU QM, MAUNY F, JOLY D, FRANCOIS S, 2015. Air pollution in moderately polluted urban areas : how does the definition of "neighborhood" impact exposure assessment?, Environmental Pollution 206, 437-448.

UNG A, WEBER C, PERRON G, HIRSCH J, KLEINPETER J, et al, 2001. Air pollution mapping over a city - virtual stations and morphological indicators. 10th International Symposium "Transport and Air Pollution", Sept., Boulder, Colorado, United States.

WORLD HEALTH ORGANISATION, 2009. Mortality and burden of disease attributable to selected major risks. http://www.who.int/healthinfo/global_burden_disease/

GlobalHealthRisks_report_full.pdf

WORLD HEALTH ORGANISATION, 2013. Outdoor Air Pollution a Leading Environmental Cause of cancer Deaths.

\section{NOTES}

1. Stations virtuelles présentant les mêmes caractéristiques que les stations réelles (mais obtenues par interpolation) - mêmes caractéristiques techniques, mêmes représentativités spatiales.

2. Les immissions représentent la charge polluante respirée (émissions après dispersion), ce sont les immissions qui ont un impact sur la santé, et qui doivent être croisées avec les données de santé.

3. Le terme advection signifie le transport d'une quantité de matière d'un élément donné (élément chimique ici) par le mouvement du milieu environnant

4. CPU : Central Proccessing Unit (temps machine) 


\section{RÉSUMÉS}

La pollution de l'air, qu'elle soit d'origine industrielle ou liée au trafic routier, a des effets reconnus sur la santé. De très nombreuses études, de par le monde, questionnent les relations pollution - santé (notamment en termes de mortalité évitable ou d'augmentation de risques respiratoires ou cardiovasculaires) que ce soit à l'échelle d'une agglomération ou d'un pays, mais peu d'entre-elles s'intéressent au processus de modélisation qui a permis de produire les données environnementales qui sont confrontées aux données médicales. Pourtant la pollution de l'air en ville ne peut se résumer à un polluant spécifique et présente une hétérogénéité spatiale (engendrée par la morphologie urbaine) qu'il est nécessaire de prendre en compte avant de pouvoir estimer un impact quantifiable sur la santé, particulièrement si l'on souhaite connaître l'exposition à court terme subie tout au long de son déplacement. Une réflexion sur la modélisation de la qualité de l'air, à échelle fine, est engagée ici. A partir de deux modèles eulériens (l'un dit «simple», l'autre dit «complexe»), nous questionnerons la faisabilité d'un modèle d'estimation en temps réel des concentrations de polluants et apporterons des éléments sur des itinéraires individuels d'exposition.

Air pollution has many recognized effects on human health. Numerous studies worldwide investigate the relationship between pollution and health, but few are interested in the modeling process that has coupled environmental data with medical data.However, measuring air pollution in the city is complicated, because it cannot be reduced to a specific pollutant (rather is consists of a mix of many pollutants). In addition, it has a spatial heterogeneity which must be taken into account before estimating impact on health, especially if one wishes to measure the effects of short-term exposure.This article aims to offer a critique on the modeling process that links environment and health,without denying the fact that air pollution has a negative impact on citizens' health. First, we discuss how air pollution is modeled. We explain why it is important to model pollutant concentrations in the air because of a lack of permanent stations making actual measurements. Then the advantages and disadvantages of the different families of deterministic models (Gaussian, Lagrangian, and Eulerian) available to improve air quality monitoring in cities are discussed.The uncertainties of those models are evaluated so as to choose the better model for understanding air pollution dispersion (immissions) in cities. Immissions are the share of pollution that must be connected with health data. This is the crux of the process because it is difficult to know the ambient air pollution levels in real time at any point in space. Gaussian models have simplified equations which mean that the concentrations are just a decreasing function of the distance (basic model). Lagrangian models are useful to follow a particle or group of particles but cannot be used if the number of components remains important (the cost of the model is proportional to the number of followed particles). Eulerian dispersion models can be used in complex urban morphology to estimate air pollutants concentrations in all places, even at a fine scale (some meters). This is the best approach to modeling based on the information of permanent stations and the computing time is independent of the number of particles followed. After validating the choice of Eulerian models which are more efficient at fine scale (a street or a district), we compared the results of a simple model and a complex model (including wind direction and velocity) from a case study: the Bonaparte street in Paris. Advantages of each model for the purpose of health impact evaluation are identified and discussed. Second, the article proposes the concept of health routes in cities so as to reduce the exposition to air pollutants in the streets, in real time. Numerous studies have shown some links between air pollution and health impacts. However, it is necessary to deepen knowledge of the pollution and to better understand individual paths (routes, medical history, and differentiated 
daily exposure). Because we spend eighty percent of our time indoors, the links between outdoor and indoor pollution must also be deepened.Uncertainties are present in all steps of the evaluation process (emissions, immissions, and individual exposure). As such, we have to be prudent when estimating risk (quantitative measurements) between pollution and health.

INDEX

Mots-clés : pollution de l'air, modélisation, modèle eulérien

\section{AUTEUR}

\section{GILLES MAIGNANT}

Chargé de Recherche CNRS - HDRUMR 912 SESSTIM-Faculté de médecine de Nice 